\section{Effect of post-consolidation regimen on symptomatic osteonecrosis in three DCOG acute Iymphoblastic leukemia protocols}

Symptomatic osteonecrosis is a serious side effect of childhood acute lymphoblastic leukemia (ALL) treatment. In affected children, the blood supply to especially the epiphysis of weight-bearing bones is insufficient. ${ }^{1}$ Osteonecrosis may resolve completely with conservative treatment or may result in debilitating long-term sequelae such as articular collapse, ultimately leading to joint replacement at an early age. ${ }^{2,3}$

Corticosteroids, used to treat ALL, largely contribute to osteonecrosis development. ${ }^{4}$ Moreover, higher cumulative corticosteroid doses may be associated with an elevated risk of osteonecrosis. A large trial from the Children's Oncology Group (CCG-1961) showed that shorter corticosteroid pulses decreased the risk of osteonecrosis despite a higher cumulative dose in adolescents. ${ }^{5}$ This strategy has been widely adopted in other pediatric ALL protocols. ${ }^{6}$ However, asparaginase has been shown to increase the risk of osteonecrosis especially when administered concurrently with corticosteroids, ${ }^{1,7}$ and its administration has been intensified in recent ALL protocols, which contributed to increased survival rates. ${ }^{8}$ The benefit of shorter pulses dexamethasone on osteonecrosis development in the context of recent asparaginase intensified regimens remains unclear.

The primary aim of this study was to compare the cumulative incidence of osteonecrosis (CION) in children treated with long (Dutch Childhood Oncology Group [DCOG] ALL-9) versus short pulses dexamethasone (asparaginase intensified ALL-10/11 medium risk group [MRG]). The secondary aim was to investigate the associations between risk factors and osteonecrosis and to assess the characteristics of patients with severe osteonecrosis.

Children aged 1-18 years with newly diagnosed ALL between January 1997 to March 2015 treated according to the DCOG ALL-9 or ALL-10/11 MRG protocol were eligible for this study. Detailed information on patient selection and data collection is provided in the Online Supplementary Appendix. For our primary aim, the CION was estimated from start post-consolidation (landmark analysis), since dexamethasone pulses started from this timepoint onwards. Consent from patients and/or legal guides for data collection had been previously obtained.

Patients were treated with dexamethasone during induction and with long pulses dexamethasone (14 days $6 \mathrm{mg} / \mathrm{m}^{2} /$ day every 7 weeks, cumulative dose non-high risk group, 1,370 $\mathrm{mg} / \mathrm{m}^{2}$; high risk group, $1,244 \mathrm{mg} / \mathrm{m}^{2}$ ) without concurrent asparaginase during post-consolidation in ALL-9 (Online Supplementary Table S1). Patients in ALL-10/11 MRG were treated with prednisone during induction and with short pulses dexamethasone (5 days $6 \mathrm{mg} / \mathrm{m}^{2} /$ day every 3 weeks, cumulative dose, 1,115 $\mathrm{mg} / \mathrm{m}^{2}$ ) with 18 or 30 weeks concurrent PEG-asparaginase $\left(2,500 \mathrm{IU} / \mathrm{m}^{2}\right.$ [ALL-10] or an individualized dose [ALL-11] every 2 weeks) during post-consolidation, resulting in six or ten dexamethasone pulses that were administered concomitantly with PEG-asparaginase.

Osteonecrosis was defined as persistent pain in joints and/or limbs (not resulting from vincristine neuropathy) developed during or in the first year after ALL treatment and confirmed by magnetic resonance imaging (and/or Xray, see the Online Supplementary Appendix). Severe osteonecrosis was defined as Ponte di Legno (PdL) grade 4 (Online Supplementary Table S2) in ALL-10/11 MRG.'

The CION since start post-consolidation therapy was estimated for patients treated in ALL-10/11 MRG versus ALL-9 using competing risk models with stem cell transplantation, second malignancy, relapse and death as competing event. In this analysis, only the subset of patients who reached post-consolidation per protocol was included. The CION since ALL diagnosis was estimated for different age categories in the total cohort. Fine and Gray's test was used to assess the difference between the CION. A univariable and multivariable Cox proportional hazard regression model was used to estimate the effect of risk factors on osteonecrosis (see the Online Supplementary Appendix for details).

Of 1,612 patients eligible for ALL-9 $(n=886)$ and ALL$10 / 11$ MRG ( $\mathrm{n}=726), 1,470(91 \%)$ were included in this study (Online Supplementary Figure S1). Thirteen hundred eighty-four (94\%) of these patients reached the start of post-consolidation therapy per protocol and were included in the landmark analysis. The baseline characteristics of patients treated in ALL-9 versus ALL-10/11 MRG were not significantly different (Online Supplementary Table S3).

In total, $79(5 \%)$ of 1,470 patients developed osteonecrosis during the study. Thirty-six $(5 \%)$ of 731 children in ALL-9 and $38(6 \%)$ of 652 children in ALL$10 / 11$ MRG who were included in the landmark analysis developed osteonecrosis, respectively. No statistically significant difference between the CION since start postconsolidation therapy for the two groups was found $(P=0.54$, Figure 1$)$; at 3 years since the start of post-consolidation therapy, the CION was $4.9 \% \quad(95 \%$ Confidence Interval $[\mathrm{CI}]=3.4-6.5)$ and $5.4 \% \quad(95 \%$ $\mathrm{CI}=3.6-7.1$ ) in ALL-9 versus ALL-10/11 MRG, respectively. In addition, the CION since ALL diagnosis was estimated, which showed no statistically significant difference $(P=0.80$, Online Supplementary Figure S2).

Table 1. Cause-specific hazard ratio estimates along with their $95 \%$ Confidence Intervals for the risk of symptomatic osteonecrosis since start post-consolidation therapy from a univariable and multivariable Cox proportional hazard regression model.

\begin{tabular}{|c|c|c|c|c|c|c|}
\hline & \multicolumn{3}{|c|}{$\begin{array}{l}\text { Univariable model } \\
\qquad n=1,383\end{array}$} & \multicolumn{3}{|c|}{$\begin{array}{l}\text { Multivariable model } \\
\qquad n=1,383\end{array}$} \\
\hline & $\mathrm{HR}_{\mathrm{cs}}$ & $95 \%$ CI & P & $\mathrm{HR}_{\mathrm{cs}}$ & $95 \%$ CI & $P$ \\
\hline $\begin{array}{l}\text { Post-consolidation regimen } \\
\text { (ALL-10/11 MRG vs. ALL-9) }\end{array}$ & 1.21 & $0.76-1.92$ & 0.416 & 0.69 & $0.43-1.11$ & 0.123 \\
\hline Age (yrs) & 1.38 & $1.30-1.46$ & $<0.001$ & 1.40 & $1.32-1.49$ & $<0.001$ \\
\hline Sex (male vs. female) & 1.18 & $0.75-1.87$ & 0.479 & 1.53 & $0.97-2.44$ & 0.070 \\
\hline BMI (SDS) & 1.01 & $0.82-1.24$ & 0.942 & 0.90 & $0.73-1.10$ & 0.289 \\
\hline
\end{tabular}

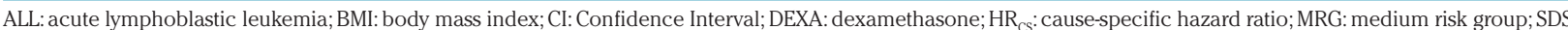
standard deviation score; yrs: years. 


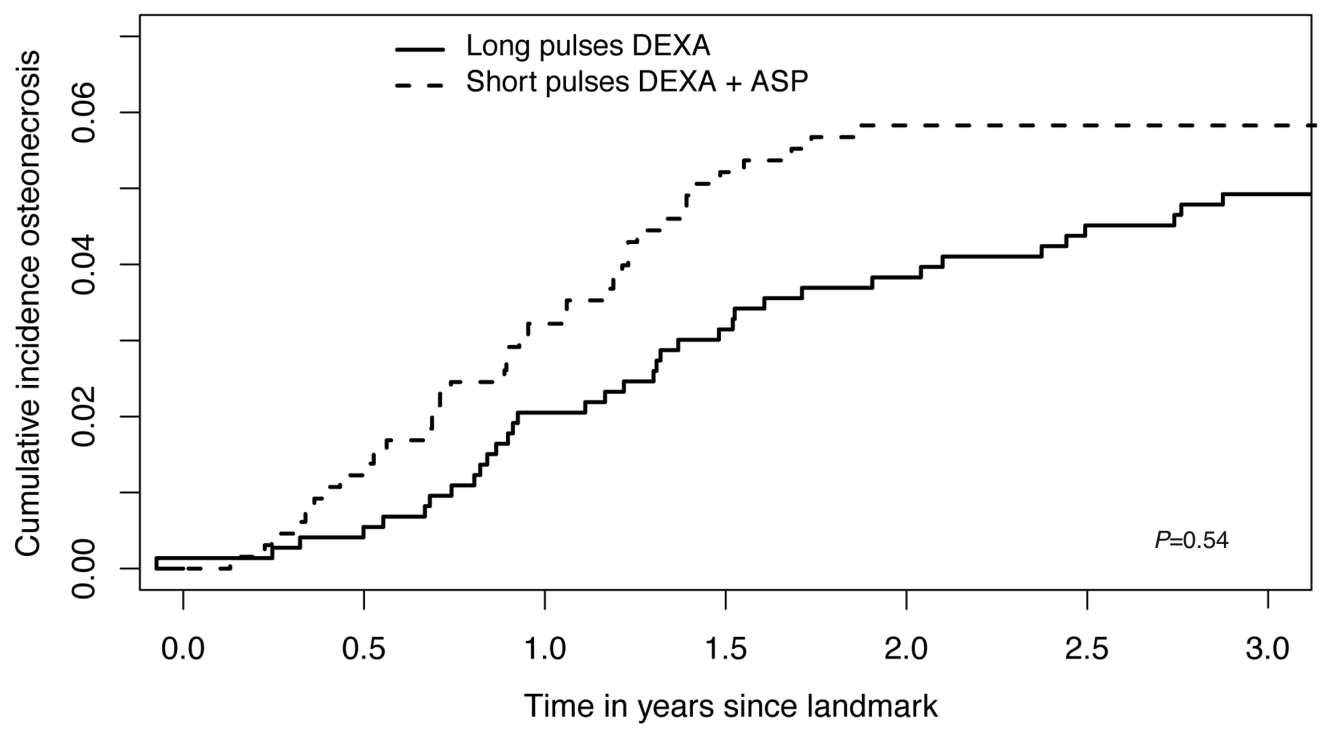

No. at risk

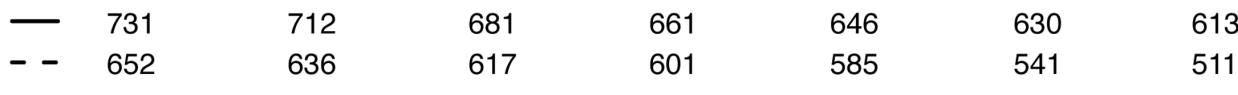

Figure 1. Cumulative incidence of symptomatic osteonecrosis for patients treated with long pulses dexamethasone ( $n=731)$ and patients treated with short pulses dexamethasone plus asparaginase $(n=652)$ since start post-consolidation therapy (landmark). ASP: asparaginase; DEXA: dexamethasone.

In multivariable analysis including type of post-consolidation treatment regimen, age at ALL diagnosis, sex and body mass index standard deviation score, age was the only significant independent risk factor (cause-specific hazard ratio $\left[\mathrm{HR}_{\mathrm{CS}}\right]=1.40 ; 95 \% \mathrm{CI}=1.32-1.50 ; P<0.001$, Table 1).

A statistically significant difference between the CION for different age categories at ALL diagnosis was observed $(P<0.001$, Figure 2$)$. At 3 years since ALL diagnosis, the CION was $1.2 \%(95 \% \mathrm{CI}=0-2.3), 14.3 \%(95 \%$ $\mathrm{CI}=10.0-18.5)$ and $31.4 \%(95 \% \mathrm{CI}=30.9-31.9 \%)$ for children aged (years) $1-9,10-14$, and 15-18, respectively.

Fifteen of 38 children (39\%) with osteonecrosis in ALL10/11 MRG experienced severe osteonecrosis. Severe osteonecrosis occurred in none of the 6 children with osteonecrosis aged (years) $1-9$, in 5 of the 15 (33\%) aged $10-14$, and in 10 of the 17 (59\%) aged 15-18.

For detailed information on the site and management of osteonecrosis, refer to the Online Supplementary Appendix.

In this study, no statistically significant difference in the CION for children treated with short versus long pulses dexamethasone was found. Based on the findings of the CCG-1961 trial, ${ }^{5}$ we hypothesized that patients treated with short pulses dexamethasone would have a lower CION. This hypothesis was consistent with a recent preclinical study which showed that asparaginase added to a discontinuous dexamethasone regimen did not increase osteonecrosis occurrence in mice. ${ }^{10}$

We realized however, that to increase the survival of children with ALL over the past decades, asparaginase intensification has played an important role. Although the combined administration of dexamethasone and asparaginase in our protocols does not allow to prove the relative contribution of asparaginase to osteonecrosis development, we think it is conceivable that intensification of ALL treatment components other than dexamethasone regimens such as asparaginase may explain our findings. There is evidence that asparaginase is associated with osteonecrosis, especially when administered concurrently with dexamethasone. ${ }^{1,7}$ We have previously shown that in patients with osteonecrosis, a hypercoagulable state may result from a lower dexamethasonerelated increase of anticoagulants in combination with a subsequent decline of these anticoagulants after asparaginase introduction. ${ }^{1}$ Furthermore, asparaginase increases the plasma concentration of dexamethasone, and in particular PEG-asparaginase may increase triglyceride levels (associated with osteonecrosis) especially in combination with dexamethasone. ${ }^{7,11,12}$ In a controlled pre-clinical model, mice receiving asparaginase plus continuous dexamethasone experienced osteonecrosis more often than those receiving dexamethasone alone.$^{13}$ Furthermore, discontinuous dexamethasone reduced the risk of osteonecrosis compared to continuous dexamethasone in the CCG-1961 trial more in patients who received intensified versus standard treatment, also suggesting that other treatment components may play a role in the effect of dexamethasone pulses duration. ${ }^{5}$ Other explanations for our finding could be that the long pulses dexamethasone in the DCOG ALL-9 protocol were already shorter than the continuous dexamethasone in the CCG-1961 trial, and administered throughout maintenance compared to during delayed intensification only, respectively.

Our results highlight the relevance of therapeutic context when interpreting results of treatment-related toxicity. Further research addressing the effect of dexamethasone and asparaginase schedules on osteonecrosis occurrence is needed. This is of interest since older children who are at highest risk of (severe) osteonecrosis are also most likely to have an unfavorable leukemia outcome, which raises serious cautiousness towards ALL treatment reduction because of toxicity. ${ }^{14}$ Furthermore, adequate treatment of osteonecrosis remains an issue: overall success of conservative treatment is limited since about $50 \%$ of patients reported persistent symptoms after 5 years of 


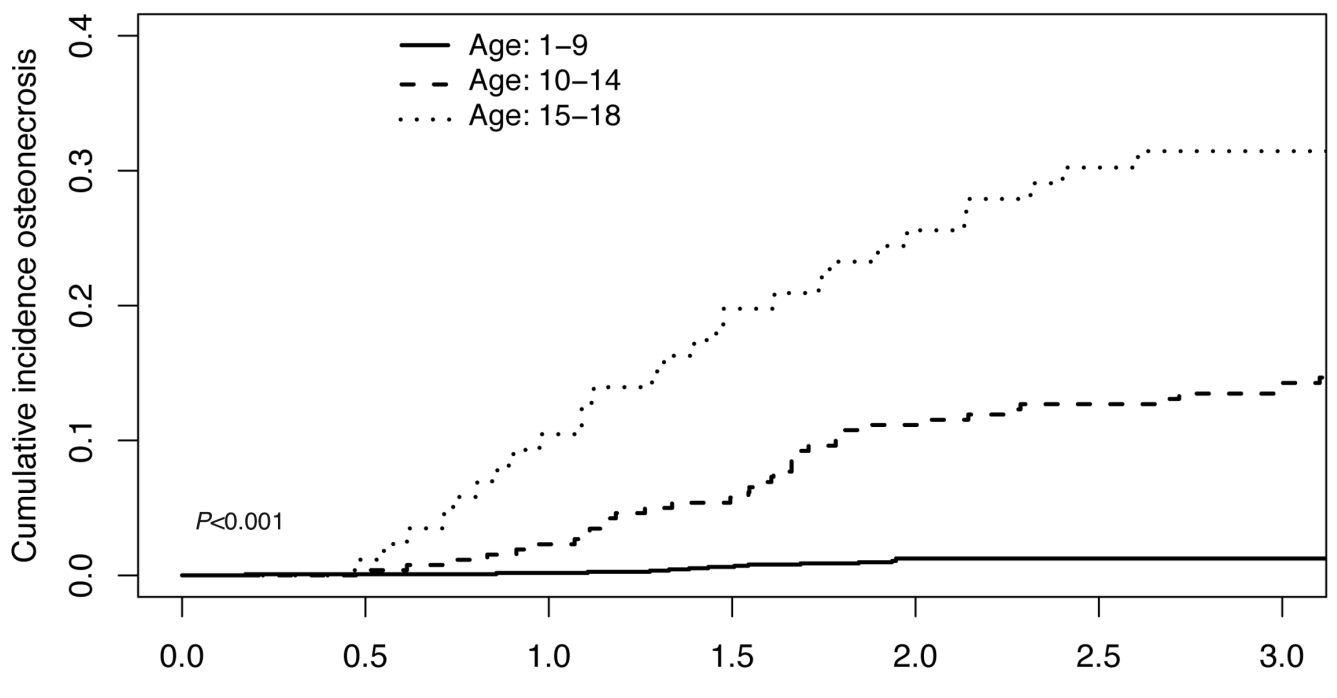

\section{No. at risk}

$\begin{array}{llllllll}- & 1124 & 1108 & 1083 & 1059 & 1040 & 1032 & 983 \\ \ldots- & 260 & 251 & 229 & 210 & 191 & 179 & 164 \\ \ldots & 86 & 84 & 70 & 61 & 53 & 47 & 39\end{array}$

Figure 2. Cumulative incidence of symptomatic osteonecrosis for children aged 1-9 years $(n=1,124), 10-14$ years $(n=260)$ and $15-18$ years $(n=86)$ since acute lymphoblastic leukemia diagnosis. ALL: acute lymphoblastic leukemia.

follow-up. ${ }^{2}$ Hence, prevention of osteonecrosis by treatment scheduling modification seems preferable since it could possibly decrease osteonecrosis associated morbidity without jeopardizing leukemia outcome.

The association between age and the risk of osteonecrosis has been thoroughly studied. Adolescents are disproportionally affected by this toxicity relative to younger children and adults. ${ }^{15}$ We here confirmed findings from other large studies, ${ }^{5,16}$ and showed in addition that the 3-year CION was significantly different in children aged (years) $1-9(1.2 \%), 10-14(14.3 \%)$, and 15-18 $(31.4 \%)$. This means that among children older than 10 years, an age group that most studies have focused on, $4,7,15$ children aged 15-18 seem to develop osteonecrosis most often, even relative to children aged 10-14 years. This is especially important since our study for the first time shows that in these 15-18-year-old patients, the osteonecrosis is more often severe. Of all patients affected by severe osteonecrosis, $67 \%$ ultimately required joint replacement and $20 \%$ still experienced chronic pain at follow-up, indicating the clinical relevance of this complication. Our finding is in line with previous studies that showed an increased risk of severe osteonecrosis and hip replacement among older children. ${ }^{5,17}$ More studies are needed to better understand the occurrence of severe and progressive osteonecrosis.

The results of our study must be interpreted in light of several limitations. We did not perform a randomized controlled trial, so differences between the cohorts other than those adjusted for may exist. We think limiting our analysis to the MRG in ALL-10/11 was justified, but could have introduced bias. However, an overall analysis comparing the CION of ALL-9 versus the entire ALL$10 / 11$ cohort showed similar results. Although we attempted to rule out differences in induction therapy by employing a landmark analysis, this protocol variation, as well as differences in asparaginase formulation, should be appreciated. Furthermore, all patients in ALL-10/11 received both short pulses dexamethasone and asparaginase, so assessing the effect of each treatment component separately was not possible.

We conclude that no statistically significant difference in the CION for children treated with short pulses dexamethasone plus asparaginase versus long pulses of dexamethasone alone during ALL post-consolidation therapy was found. We postulate that the protective effect of shorter pulses dexamethasone on osteonecrosis occurrence may be attenuated by recent intensification of other treatment components such as asparaginase. Among children older than 10 years, especially children aged 15-18 years developed symptomatic, in particular severe osteonecrosis.

Jenneke E. van Atteveld, Hester A. de Groot-Kruseman, ${ }^{2}$ Marta Fiocco, ${ }^{1,3,4}$ Maarten H. Lequin, ${ }^{1,5}$ Sebastian J.C.M.M. Neggers, ${ }^{1}$ Saskia M.F. Pluijm, ${ }^{1}$ Inge M. van der Sluis, ${ }^{1}$ Rob Pieters ${ }^{1}$ and Marry M. van den Heuvel-Eibrink ${ }^{1}$

${ }^{1}$ Princess Máxima Center for Pediatric Oncology, Utrecht; ${ }^{2}$ Dutch Childhood Oncology Group (DCOG), Utrecht; ${ }^{3}$ Medical Statistics Unit, Department of Biomedical Data Science, Leiden University Medical Center, Leiden; ${ }^{4}$ Mathematical Institute, Leiden University, Leiden and ${ }^{5}$ Department of Radiology, University Medical Center Utrecht, Utrecht, the Netherlands

Correspondence: JENNEKE E. VAN ATTEVELD

j.e.vanatteveld@prinsesmaximacentrum.nl

doi:10.3324/haematol.2020.257550

Disclosures: no conflicts of interest to disclose.

Contributions: JEvA study design, data collection, data analysis, data interpretation, manuscript writing; $H A d G-K$ data collection, data analysis, data interpretation, manuscript writing; MF data analysis, data interpretation, manuscript writing; MHL data interpretation, manuscript writing; SJCMMN data interpretation, manuscript writing; SMFP data interpretation, manuscript writing; 
$I M v d S$ data interpretation, manuscript writing; RP study design, data interpretation, manuscript writing; MMvdH-E study design,

data interpretation, manuscript writing.

\section{References}

1. te Winkel ML, Appel IM, Pieters R, van den Heuvel-Eibrink MM Impaired dexamethasone-related increase of anticoagulants is associated with the development of osteonecrosis in childhood acute lymphoblastic leukemia. Haematologica. 2008;93(10):1570-1574.

2. te Winkel ML, Pieters R, Hop WCJ, et al. Prospective study on incidence, risk factors, and long-term outcome of osteonecrosis in pediatric acute lymphoblastic leukemia. J Clin Oncol. 2011;29(31):41434150.

3. Mattano LA, Sather HN, Trigg ME, Nachman JB. Osteonecrosis as a complication of treating acute lymphoblastic leukemia in children: a report from the Children's Cancer Group. J Clin Oncol. 2000; 18(18):3262-3272

4. Girard P, Auquier P, Barlogis V, et al. Symptomatic osteonecrosis in childhood leukaemia survivors: prevalence, risk factors and impact of quality of life in adulthood. Haematologica. 2013;98(7):1089-1097.

5. Mattano LA, Devidas M, Nachman JB, et al. Effect of alternate-week versus continuous dexamethasone scheduling on the risk of osteonecrosis in paediatric patients with acute lymphoblastic leukaemia: results from the CCG-1961 randomised cohort trial Lancet Oncol. 2012;13(9):906-915.

6. Toft N, Birgens H, Abrahamsson J, et al. Results of NOPHO ALL2008 treatment for patients aged 1-45 years with acute lymphoblastic leukemia. Leukemia. 2018;32(3):606-615.

7. Kawedia JD, Kaste SC, Pei D, et al. Pharmacokinetic, pharmacodynamic, and pharmacogenetic determinants of osteonecrosis in children with acute lymphoblastic leukemia. Blood. 2011;117(8):23402347; quiz 2556

8. Pieters R, Hunger SP, Boos J, et al. L-asp treatment in ALL: a focus on erwinia. Cancer. 2011:117(2):238-249.
9. Schmiegelow K, Attarbaschi A, Barzilai S, et al. Consensus definitions of 14 severe acute toxic effects for childhood lymphoblastic leukaemia treatment: a Delphi consensus. Lancet Oncol. 2016;17(6):e231-e239.

10. Karol SE, Janke LJ, Panetta JC, et al. Asparaginase combined with discontinuous dexamethasone improves antileukemic efficacy without increasing osteonecrosis in preclinical models. PLoS One. 2019;14(5):e0216328.

11. Yang L, Panetta JC, Cai X, et al. Asparaginase may influence dexamethasone pharmacokinetics in acute lymphoblastic leukemia. J Clin Oncol. 2008:26(12):1932-1939.

12. Finch ER, Smith CA, Yang W, et al. Asparaginase formulation impacts hypertriglyceridemia during therapy for acute lymphoblastic leukemia. Pediatr Blood Cancer. 2019;(Aug 2019):1-10.

13. Liu C, Janke LJ, Kawedia JD, et al. Asparaginase potentiates glucocorticoid-induced osteonecrosis in a mouse model. PLoS One 2016;11(3):1-13.

14. Pieters R, de Groot-kruseman H, Van Der Velden V, et al. Successful therapy reduction and intensification for childhood acute lymphoblastic leukemia based on minimal residual disease monitoring: study ALL10 from the Dutch Childhood Oncology Group. J Clin Oncol. 2016;34(22):2591-2601.

15. Toft N, Birgens H, Abrahamsson J, et al. Toxicity profile and treatment delays in NOPHO ALL2008-comparing adults and children with Philadelphia chromosome-negative acute lymphoblastic leukemia. Eur J Haematol. 2016;96(2):160-169.

16. Möricke A, Zimmermann M, Valsecchi MG, et al. Dexamethasone vs prednisone in induction treatment of pediatric ALL: results of the randomized trial AIEOP-BFM ALL 2000. Blood. 2016;127(17):21012112 .

17. Mogensen SS, Harila-Saari A, Makitie O, et al. Comparing osteonecrosis clinical phenotype, timing, and risk factors in children and young adults treated for acute lymphoblastic leukemia. Pediatr Blood Cancer. 2018;65(10):e27300. 